\title{
CONFORMAL GEOMETRY AND COMPLETE MINIMAL SURFACES
}

\author{
ROB KUSNER
}

1. Introduction. This note applies new ideas from conformal geometry to the study of complete minimal surfaces of finite total curvature in $R^{3}$. The two main results illustrate this in complementary ways. Theorem A implies several uniqueness or nonexistence corollaries, while Theorem B constructs new examples, including the first complete immersed nonorientable minimal surfaces with finite total curvature and embedded ends. This construction is based upon the close relationship between these minimal surfaces and certain compact immersed surfaces minimizing the conformally invariant functional $W=\int H^{2} d a$ (Theorems $\mathrm{C}$ and $\mathrm{D}$ ).

2. Main results. Let $M \ell R^{3}$ be an immersed complete minimal surface with finite total curvature $c(M)=\int_{M} K d a$. Then (reinterpreting classical results of [13]) there is associated to $M$ a $C^{1, \alpha}$ compact (possibly branched at $\infty$ ) immersed surface $\bar{M} \ell S^{3} \sim R^{3} \cup \infty$ such that $\bar{M}-\infty \sim M$. (The conformal equivalence $S^{3} \sim R^{3} \cup \infty$ is via stereographic projection.)

Denote by $\mu_{x}$ the number of sheets of $\bar{M}$ through a point $x \in S^{3}$, and set $\mu(M)=\max \left\{\mu_{x} \mid x \in R^{3}\right\}$, and $\eta(M)=\mu(\infty)$. Observe that $\eta(M)$ equals the number of ends of $M$ iff each end is embedded iff $\bar{M} \ell S^{3}$ is unbranched [9].

THEOREM A. $\mu(M) \leq \eta(M)$; equality implies $M$ is a union of planes.

COROLlary 1. (i) If $\eta(M)=1$, then $M$ is a plane [7].

(i) If $\eta(M)=2$ and $M$ is connected, then $M$ is embedded. (This permits a simpler proof that $M$ is a catenoid [14].)

Assume now that $M$ is connected and that each end is embedded. Express each end as $g r a p h(h)$ where $h \simeq c r^{2} \log r$ is the height over its tangent plane at $\infty$ (in terms of inverted polar coordinates). An end is flat if $c=0$; otherwise, it is a catenoid end. By viewing $\infty$ as a triple-point of $\bar{M} \ell S^{3}$ (called transverse if each small perturbation of $\bar{M}$ also has a triple-point) and using the relation [1] between the Euler number $\chi(\bar{M})$ and the number of triple-points, deduce

COROLlaRY 2. If $\eta(M)=3$, then either

(i) $\chi(\bar{M})$ is even and the ends of $M$ are not transverse; or

(ii) $\chi(\bar{M})$ is odd and the ends of $M$ are transverse.

Received by the editors August 18, 1986 and, in revised form, March 31, 1987.

1980 Mathematics Subject Classification (1985 Revision). Primary 53A10, 49F10, 57R42.

Research partially supported by NSF Grant MCS 81-23356 and by a Regents' Fellowship at the University of California. Research impartially supported by Tiny. 
(If $M$ has a catenoid end, then an argument using the balancing formula $[\mathbf{8}, \mathbf{1 7}]$ shows that the ends cannot be transverse; so, in (ii), all ends must be flat.)

Alternative (i) occurs in different ways: compare the trinoid [7] and the skirted catenoids $[\mathbf{4}, \mathbf{6}]$. Alternative (ii) is also possible, as the first of the following new examples demonstrates.

THEOREM B. For every odd integer $p \geq 3$ there exists an immersed complete minimal surface $M_{p} \ell R^{3}$ with finite total curvature $c\left(M_{p}\right)=-2 \pi(2 p-1)$, and with $\eta\left(M_{p}\right)=p$ embedded flat ends. The associated compact surface $\bar{M}_{p} \sim R P^{2}$, so $M_{p}$ is nonorientable. $M_{p}$ contains $p$ straight lines which lie in a plane and meet at equal angles. The dihedral group of order $2 p$ acts on $M_{p}$ by reflections around these lines.

The asserted properties of $M_{p}$ can be explicitly verified by checking through the following.

REMARK 1. (i) In terms of the meromorphic coordinate $z$ on the universal cover $\widetilde{R P}^{2} \sim S^{2}$, the Weierstrass representatives of $M_{p}$ are (cf. $\left.[10,12,15]\right)$

$$
g_{p}(z)=\frac{z^{p-1}\left(z^{p}-s\right)}{\left(s z^{p}+1\right)}, \quad f_{p}(z)=\frac{i\left(s z^{p}+1\right)^{2}}{\left(z^{2 p}+r z^{p}-1\right)^{2}}
$$

where $s=\sqrt{2 p-1}$ and $r=2 s /(p-1)$.

(ii) The Weierstrass forms $\varphi=f\left(1-g^{2}, i\left(1+g^{2}\right), 2 g\right) d z$ are exact, so $M_{p}$ is represented by the conformal harmonic map $\operatorname{Re} \Phi_{p}$, where

$$
\Phi_{p}(z)=\int^{z} \varphi_{p}=\frac{i}{z^{2 p}+r z^{p}-1}\left(z^{2 p-1}-z,-i\left(z^{2 p-1}+z\right), \frac{p-1}{p}\left(z^{2 p}+1\right)\right) .
$$

From this formula it follows that $M_{p}$ is immersed and that each end is flat and embedded.

(iii) The symmetries of $M_{p}$ are expressed by the following identities.

$$
\Phi_{p}\left(z_{*}\right)=\overline{\Phi_{p}(z)}, \quad \Phi_{p}(\bar{z})=\overline{\Phi_{p}(z)} A, \quad \Phi_{p}\left(e^{2 \pi i / p} z\right)=\Phi_{p}(z) B^{2},
$$

where $z_{*}=-1 / \bar{z}$,

$$
A=\left(\begin{array}{ccc}
-1 & 0 & 0 \\
0 & 1 & 0 \\
0 & 0 & -1
\end{array}\right), \quad \text { and } \quad B=\left(\begin{array}{ccc}
\cos \pi / p & -\sin \pi / p & 0 \\
\sin \pi / p & \cos \pi / p & 0 \\
0 & 0 & 1
\end{array}\right) \text {. }
$$

REMARK 2 . For even $p \geq 2$, the same formula for $\Phi$ gives a complete minimally immersed $N_{p} \ell R^{3}$ with $\eta\left(N_{p}\right)=2 p$ embedded flat ends, total curvature $c\left(N_{p}\right)=-4 \pi(2 p-1)$, and $\bar{N}_{p} \sim S^{2}$. The identities

$$
\Phi_{p}(\bar{z})=\overline{\Phi_{p}(z)} A \text { and } \Phi_{p}\left(e^{\pi i / p} z_{*}\right)=\overline{\Phi_{p}(z)} B
$$

show that $N_{p}$ has dihedral symmetry of order $4 p$.

It has been an outstanding question to determine the possible values $c(M)$ for the total curvature of a complete minimal surface $M \ell R^{3}$ [13]. The GaussBonnet formula implies that $c(M)$ is a negative multiple of $2 \pi$, and the value $-2 \pi$ is forbidden [12] (see also [13]). These new examples $M_{p}$ can be deformed 
into complete minimal Möbius strips with total curvature $-2 \pi p$ (cf. [12] for $p=3$ ); together with the plane, catenoid,.,$n$-oid $[\mathbf{7}], \ldots$, they show that all possible values are attained.

COROLlaRY 3. For every integer $j \geq 0$, except $j=1$ (which is prohibited), there exists a complete immersed minimal surface $M \ell R^{3}$ with $c(M)=-2 \pi j$.

3. Lemmas from conformal geometry. Let $M^{\prime} \ell R^{3}$ be a compact surface with $\mu\left(M^{\prime}\right)=\mu_{x}=p$. Recall that the work (or Willmore functional [2]) is defined by

$$
W\left(M^{\prime}\right)=\int_{M^{\prime}} H^{2} d a,
$$

where $H$ is the mean curvature of $M^{\prime} \ell R^{3}$.

LEMMA 1. $W\left(M^{\prime}\right) \geq 4 \pi p[\mathbf{1 1}]$; equality holds iff there is a conformal automorphism $G$ of $S^{3}$ with $G(x)=\infty$ such that $M \sim G\left(M^{\prime}\right)-\infty$ is a complete minimal surface in $R^{3}$ with $\eta(M)=p$.

ProOF. Note that $\chi\left(M^{\prime}\right)=\chi(M)+\eta(M)$. By the conformal invariance of the density $\left(H^{2}-K\right) d a$, and by the Gauss-Bonnet formulas, compute

$$
\begin{aligned}
W\left(M^{\prime}\right) & =\int_{M^{\prime}}\left(H^{2}-K\right) d a+\int_{M^{\prime}} K d a \\
& =\int_{M}\left(H^{2}-K\right) d a+2 \pi \chi\left(M^{\prime}\right) \\
& =\int_{M} H^{2} d a-2 \pi(\chi(M)-\eta(M))+2 \pi(\chi(M)+\eta(M)) \\
& =\int_{M} H^{2} d a+4 \pi \eta(M) \\
& \geq 4 \pi p,
\end{aligned}
$$

with equality iff $H \equiv 0$ on $M$.

Next, define the conformal tangent bundle $C T M^{\prime}$ of $M^{\prime} \ell S^{3}$ to be the $S^{2}$ bundle over $M^{\prime}$ whose fibre over a point $x$ is the unique round sphere in $S^{3}$ tangent to- and with the same mean curvature as- $M^{\prime}$ at $x$.

LEMMA 2. Suppose there are distinct points $x_{1}, x_{2}$ in $R^{3}$ and conformal automorphisms $G_{1}, G_{2}$ of $S^{3}$ such that $G_{k}\left(x_{k}\right)=\infty$ and $M_{k} \sim G_{k}\left(M^{\prime}\right)-\infty$ is a complete minimal surface in $R^{3}$ for $k=1,2$. Then $M^{\prime}$ is a union of round spheres, and each $M_{k}$ is a union of planes.

Proof. Observe that each $G_{k}$ maps $C T M^{\prime}$ to the (ordinary) tangent bundle of $M_{k}$. But every tangent plane of (say) $M_{2}$ meets $\infty$, so every tangent plane of the minimal surface $M_{1}$ meets the point $x=G_{1} G_{2}^{-1}(\infty)=G_{1}\left(x_{2}\right) \neq$ $\infty$. This implies $M_{1}$ is a minimal cone over $x$, i.e. a union of planes, proving the lemma.

Now to prove Theorem $\mathrm{A}$, in case each end of $M$ is embedded, use Lemma 1 to rule out $\mu(M)>\eta(M)$, and Lemma 2 to treat the case $\mu(M)=\eta(M)$. If the ends are not embedded, one can still apply these lemmas for branched 
immersed surfaces, with minor changes in the proofs (e.g. the Gauss-Bonnet formula; see [9]).

NOTE. Recently D. Hoffman and W. Meeks have proved the first part of Theorem A using the monotonicity formula for area and quadratic area growth [16]. It is interesting to note that Lemma 1 is closely related to monotonicity of volume for the 3-cone over $M^{\prime}$ in $R^{4}$ as well [8]; indeed, Lemmas 1 and 2 together may be viewed as a conformal monotonicity formula.

4. Static surfaces and minimal surfaces. Let $\bar{M} \ell S^{3}$ be the compact immersed surface associated to a complete minimal surface $M \ell R^{3}$ with finite total curvature and embedded flat ends. Let $M^{\prime} \ell R^{3}$ be obtained from $\bar{M}$ by a conformal automorphism of $S^{3}$. Then one can show that $M^{\prime}$ is a static surface, i.e. a critical point for the work $W=\int H^{2} d a$, i.e. a solution to the fourth-order partial differential equation $\Delta H+2\left(H^{2}-K\right) H=0$.

It is a beautiful result of $\mathrm{R}$. Bryant that, provided $M^{\prime} \sim S^{2}$, the converse is true [2]. Since $R P^{2}$ is double covered by $S^{2}$, this is also the case when $M^{\prime} \sim R P^{2}$. Hence Theorem $\mathrm{B}$ is equivalent to

THEOREM C. For every odd integer $p \geq 3$ there exists a static surface $M_{p}^{\prime} \ell R^{3}$ with $M_{p}^{\prime} \sim R P^{2}$ and $W\left(M_{p}^{\prime}\right)=4 \pi p . M_{p}^{\prime}$ has (conformal) dihedral symmetry of order $2 p$, and minimizes the work $W$ among all $R P^{2} \ell R^{3}$ with this symmetry.

REMARK 3. (i) Originally the author [8] constructed these static $M_{p}^{\prime}$ variationally, and used the symmetries to deduce the Weierstrass representation for the minimal surfaces $M_{p} \ell R^{3}$.

(ii) The static $N_{p}^{\prime} \sim S^{2}$ associated to the complete minimal surface $N_{p}$ for even $p$ has a variational characterization as well: $N_{p}^{\prime}$ is the $W$-minimax surface for symmetric eversions $[\mathbf{5}, \mathbf{8}]$ of $S^{2}$ in $R^{3}$.

The Willmore problem [9] for immersed $R P^{2} \ell R^{3}$ can now be solved.

COROLlaRY 4. The Boy's surface $M_{3}^{\prime} \ell R^{3}$ achieves the minimum possible value $W=12 \pi$ of the work among all immersed $R P^{2} \ell R^{3}$.

This follows from Lemma 1 and the fact that any $R P^{2} \ell R^{3}$ has a triplepoint.

REMARK 4. The author has used $M_{3}^{\prime}$, together with area estimates for minimal surfaces in $S^{3}$ and simple geometric constructions, to establish the strict inequalities

$$
\inf W(M)<\left\{\begin{array} { l } 
{ 8 \pi } \\
{ 1 2 \pi } \\
{ 1 6 \pi }
\end{array} \quad \text { for compact } \left\{\begin{array}{l}
\text { orientable } \\
\text { nonorientable, } \chi \text { even } \\
\text { nonorientable }, \chi \text { odd }
\end{array}\right.\right.
$$

immersed surfaces $M \ell R^{3}$, as well as strict upper bounds for the infimum of $W$ over a given regular homotopy class [9].

5. Moduli. By deforming the Gauss map $g$ for the corresponding minimal surface, one can show that (for odd $p$ ) the moduli space $\Pi_{p}$ of static $R P^{2} \ell R^{3}$ with $W=4 \pi p$ (modulo conformal automorphisms) is a noncompact complex 
variety of dimension $p-2$. (An easier argument shows that the dimension of the moduli space $\Sigma_{p}$ of static spheres with $W=8 \pi p$ is $2 p-2$.) These new examples (Theorem C) are the "most symmetric" static $R P^{2} \ell R^{3}$.

'THEOREM D. $M_{p}^{\prime}$ is the unique surface in $\Pi_{p}$ with (conformal) dihedral symmetry of order $2 p$.

Compactification of $\Pi_{p}$ yields interesting complete minimal surfaces. For example, R. Bryant [3] has shown $\Pi_{3} \cong$ a closed half-plane in $C$, and that the minimal Möbius strip [12] provides a natural one-point compactification to $a$ closed disk. (Bryant also has computed $\Sigma_{2}$ explicitly [2].)

The author wishes to thank Robert Bryant, David Hoffman, Bill Meeks and Rick Schoen for their comments.

\section{REFERENCES}

1. T. Banchoff, Triple points and surgery of immersed surfaces, Proc. Amer. Math. Soc. 46 (1974), 407-413.

2. R. Bryant, A duality theorem for Willmore surfaces, J. Differential Geom. 20 (1984), 23-53.

3.

4. C. Costa, Imersões minimas completas em $R^{3}$ de genero um e curvatura total finita, Doctoral thesis, IMPA, Rio de Janeiro, Brasil, 1982.

5. G. Francis, Some equivariant eversions of the sphere (privately circulated manuscript), 1977.

6. D. Hoffman and W. Meeks III, Complete embedded minimal surfaces of finite total curvature, Bull. Amer. Math. Soc. (N.S.) 12 (1985), 134-136.

7. L. Jorge and W. Meeks III, The topology of complete minimal surfaces of finite total Gaussian curvature, Topology 22 (1983), 203-221.

8. R. Kusner, Complete minimal surfaces by minimizing $\int H^{2}$ (lecture notes), 1984.

9. __ Comparison surfaces for the Willmore problem, Pacific J. Math. (to appear).

10. H. B. Lawson, Lectures on minimal submanifolds. I, Publish or Perish, Berkeley, 1980.

11. P. $\mathrm{Li}$ and S. T. Yau, $A$ new conformal invariant and its applications to the Willmore conjecture and the first eigenvalue of compact surfaces, Invent. Math. 69 (1982), 269-291.

12. W. Meeks III, The classification of complete minimal surfaces in $R^{3}$ with total curvature greater than $-8 \pi$, Duke Math. J. 48 (1981), 523-535.

13. R. Osserman, Global properties of complete minimal surfaces in $E^{3}$ and $E^{n}$, Ann. of Math. (2) 80 (1964), 340-364.

14. R. Schoen, Uniqueness, symmetry and embeddedness of minimal surfaces, J. Differential Geom. 18 (1983), 791-809.

15. M. Spivak, A comprehensive introduction to differential geometry. IV, Publish or Perish, Berkeley, 1975.

16. D. Hoffman and W. Meeks III, The classical theory of minimal surfaces (in preparation).

17. N. Korevaar, R. Kusner and B. Solomon, The structure of complete embedded surfaces with constant mean curvature (preprint), 1987.

\section{Department of Mathematics, University of California, Berkeley, CALIFORNIA 94720}

Current address: Department of Mathematics, University of California, San Diego, La Jolla, California 92093 